Laser Chem. 1988, Vol. 9, pp. 107-122

(C) 1988 Harwood Academic Publishers GmbH

Photocopying permitted by license only

Reprints available directly from the Publisher

Printed in the United Kingdom

\title{
Interdependence of Optical Excitation and Surface Chemistry in Laser Induced Deposition and Etching
}

\author{
F. A. HOULE \\ IBM Research Division, Almaden Research Center, 650 Harry Road, \\ San Jose, CA 95120-6099
}

(Received January 20, 1988)

\begin{abstract}
Laser-assisted modification of solid surfaces, e.g. thin film deposition and etching, frequently involves a chemically reacting system whose optical and thermal properties change continuously in time. This leads to a coupling between excitation and reaction which has profound consequences for the cumulative effect of microscopic processes occurring at the surface, whether thermal or photochemical in nature. An understanding of this coupling provides an essential bridge between investigations of discrete mechanistic steps in surface photoreactions and measurements of macroscopic rates of laser etching and deposition. A range of situations have been found to lead to significant interdependence between optical properties and reactivity: formation of waveguides and gratings, chemical enhancement of absorption, coupling of optical properties and temperature, and optical feedback in scanning. Typical examples are presented and discussed. The real-time effects of growth of a reflecting $\mathrm{Ni}$ film on an absorbing substrate, of optical interference in the laser thermal oxidation of copper and of grating formation in the photochemical deposition of copper are described in detail.
\end{abstract}

KEY WORDS: Laser etching and deposition; solid surfaces.

\section{INTRODUCTION}

The ability to modify solid surfaces on a highly localized scale, typically of the order of microns, is probably the single most attractive feature of laser chemical processing in technological applications. Activity in this 
area has been particularly intense since 1978, and hundreds of papers demonstrating deposition and etching of various elements and compounds and optimization of processes for specific purposes have been published. ${ }^{1-6}$ Significant advances have been made toward obtaining materials of high quality using a minimum of processing steps. Many workers have taken advantage of unique characteristics of the laserparticularly spatial coherence and, in the case of pulsed lasers, high fluences during short irradiation times-to achieve specific results. Studies of the basic chemistry and physics of surface photoreactions important in etching and deposition have also appeared. ${ }^{1,4}$ While their relevance is clear, it is sometimes difficult to associate the results directly with data on etch rates, for example, or the resistivities of deposited films. One part of the information needed to connect these two types of studies is a thorough understanding of the variability of optical properties of the solid surface as it is undergoing modification, and of local temperature if laser excitation or the chemical reaction itself results in heating. Such considerations are particularly important in laser deposition of thin films since the optical and thermal properties of the substrate are often quite different from those of the growing film, and the temperature dependence of the thermal conductivity and the complex index of refraction can led to continuously varying chemistry. In some cases steady state is never reached. Despite the intricate nature of the problem, significant progress has been made in identification of systems and optical configurations where real-time characteristics of surface and substrate must be considered. Several examples are selected and discussed in this paper. For purposes of comparison, they have been classified into three groups: monotonic changes in absorption, interactions leading to periodic behavior, and scattering and channelling of light. In some cases a full theoretical analysis of the experimental data is available, allowing specific observations to be generalized to a range of situations.

\section{MONOTONIC CHANGES IN OPTICAL ABSORPTION}

As film grows or a substrate etches during surface illumination, chemical rections induced by the laser can modify the absorbance of the system in real time. This can lead to enhanced or reduced rates of reaction, depending on whether the amount of light absorbed during 
some time interval increases or decreases. ${ }^{7-9}$ The first quantitative analysis of this type of effect was reported for the case of $\mathrm{Ni}$ deposition on $\mathrm{SiO}_{2}$ using an infrared laser. ${ }^{8,9}$ The growing $\mathrm{Ni}$ film is more reflecting than the substrate, causing the efficiency of laser heating at constant power to diminish. The surface temperature initially increases as the system equilibrates, then decreases as the optical properties of the Ni film start to dominate absorption (at a Ni film thickness of a few tens of Angstroms). The thermal decomposition rate, comprising temperature dependent steps of sticking, decomposition and desorption, varies accordingly, eventually reaching a slower, steady-state level. Data for $\mathrm{W}$ deposition from $\mathrm{WF}_{6}$ show more extreme behavior: the increase in reflectivity is sufficient to lower the film temperature to the point where the film stops growing. The limiting thickness is found to be several thousand Angstroms under the experimental conditions used. These qualitative arguments concerning the origin of self-limiting effects are supported by a quantitative, theoretical description of the experimental data. ${ }^{9}$ The growing film is modelled by a time-dependent neutral density filter at the same temperature as the $\mathrm{SiO}_{2}$ substrate. The film growth kinetics are assumed to be dominated by decomposition at the surface. A parameterized correction for gas transport is included. Peak growth rates and film shapes are obtained from the calculated time-dependent deposit thicknesses and widths for comparison with experiment. Despite several approximations, such as neglect of spatial variations in reactant gas density above the deposit and of nucleation kinetics, and assumption of steady-state chemical kinetics for film growth, the experimental data and theory agree within a factor of two below $2 \mathrm{~W}$.

Many of the same considerations apply when the beam is scanned over the surface. A local increase in reflectivity can lead to nonuniform film thicknesses, and even breaks in deposited lines if the substrate is not composed of a single material. For example, $\mathrm{W}$ lines deposited as connectors between pre-existing $\mathrm{W}$ lines are discontinuous unless the substrate reflectance is monitored in real time and the laser power increased to compensate for decreased absorption. ${ }^{10}$

Analogous variations in optical absorption have been observed in polymer etching using excimer lasers. ${ }^{11-13}$ As the incident fluence is increased above the threshold for polymer ablation, increasing quantities of absorbing material (gases and particulates) are ejected from the surface during the laser pulse. The ejected plume acts as a time- 
dependent neutral density filter, directly affecting the amount of laser light reaching the surface and hence the amount of material removed by each laser pulse. For a given type of polymer, the physical nature of the attenuator and hence the amount of light actually reaching the polymer film can depend on laser fluence. Transmission measurements of polymethylmethacrylate (PMMA) films during an excimer laser pulse at 248,222 and $193 \mathrm{~nm}$ show $50 \%$ or more attenuation of the beam at fluences several times threshold. ${ }^{11}$ Closer to threshold, redeposition of debris and surface roughening leads to considerable scattering of the incident pulse.

\section{PERIODIC BEHAVIOR}

The systems described in the preceding section all exhibit a direct relationship between the rate of reaction and the instantaneous optical properties of the system. Additional factors besides a monotonic change in absorption can be involved in the interaction between excitation and reaction, however. This can be manifested in a variety of ways, perhaps the most interesting of which is periodic behavior. For example, a time-dependent change in the temperature of the system which is only indirectly related to the optical properties of the system can act independently of them to influence a surface reaction. If a laser is being used to deposit a line by heating the substrate, the deposit can act as a heat sink for the irradiated zone, leading to lower steady-state temperatures than are present when the line is initiated. ${ }^{14}$ For specific combinations of reflectivity, thermal conductivity, and scan rate, the stripe can cool the reaction zone to the point where deposition ceases, and does not resume until the laser spot has moved sufficiently far from the existing deposit for the rise in temperature to allow film growth to start again. This leads to stripes which resemble a string of beads rather than a ribbon. ${ }^{10,15}$ A strong temperature dependence of the chemical composition (and hence optical properties) of the deposit and/or the substrate can also result in periodic structures during scanning. They arise because the composition of the leading edge of a line is directly affected by the composition, i.e. optical absorbance and temperature, of the region immediately behind it, and in turn will affect the area in front of it. Several systems exhibiting cyclic coupling between local metal concentration and extent of sub- 
strate heating by the laser have been identified: deposition of $\mathrm{Fe}$ lines from $\mathrm{Fe}(\mathrm{CO})_{5},{ }^{16}$ and $\mathrm{Pd}$ from a spun-on metallo-organic film. ${ }^{17}$ Highly exothermic film growth initiated by laser heating can exhibit formally similar behavior. ${ }^{18}$

Periodic behavior is also found during stationary irradiation when the growing film acts as an interference filter, resulting in alternating periods of constructive and destructive inteference, and, correspondingly, low and high absorption of the incident laser beam. A good example of such a system is the laser-driven thermal oxidation of a $5000 \AA$ thick copper film supported on a sapphire substrate, which has been studied both experimentally and theoretically. ${ }^{19,20}$ It has been observed that the rapid rate of laser thermal oxidation is frequently much faster than furnace oxidation, and suggestions were made that this is due variously to stresses engendered in the film during the reaction, photo-enhanced diffusion and time-dependent optical coefficients, depending on the details of the particular optical source used. ${ }^{19}$ As a result, conventional furnace oxidation kinetics (under which the rate is limited by solid state diffusion ${ }^{21}$ ) were not believed to be applicable when an optical heat source is used. To test these ideas, and to learn as much as possible about the real-time behavior of laser thermal oxidation of copper, the reflectance of the copper-copper oxide system was measured during oxide growth at two wavelengths, as illustrated in Figure 1a. One of the probe beams was split off from the $\mathrm{cw} \mathrm{Ar}^{+}$beam driving the oxidation reaction. The second, from a $\mathrm{HeNe}$ laser, was chosen such that the two bracketed a change in optical properties of both the metal and its oxide. The purpose of this experimental approach was two-fold: to observe the interaction of the driving beam with the substrate, and to ensure an accurate interpretation of the data by requiring an analysis of two independent data sets for each set of experimental conditions. The reflectance data showed strong oscillations during laser oxidation, as seen in Figure 1b. Since $\mathrm{CuO}$ is not transparent enough to sustain interference at the probe wavelengths used $(514.5 \mathrm{~nm}$ and $632.8 \mathrm{~nm})$ these data are an immediate indication that the phase of the oxide formed is $\mathrm{Cu}_{2} \mathrm{O}$. The assignment is consistent with data from Auger electron spectroscopy and depth profiling.

Optical interferometry is a classical method for determining position and motion of an interface, and for an initial analysis of the data it is tempting to use the proportionality between interference minima or 
(a)

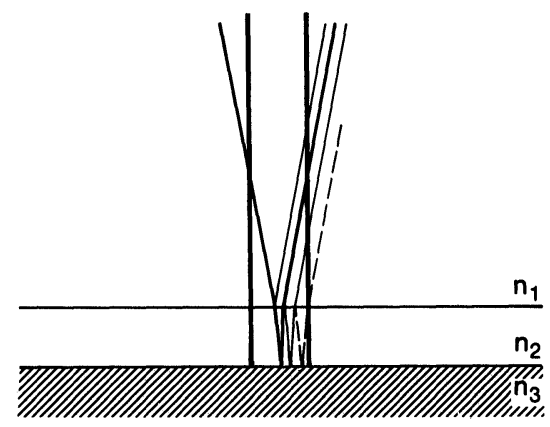

(b)

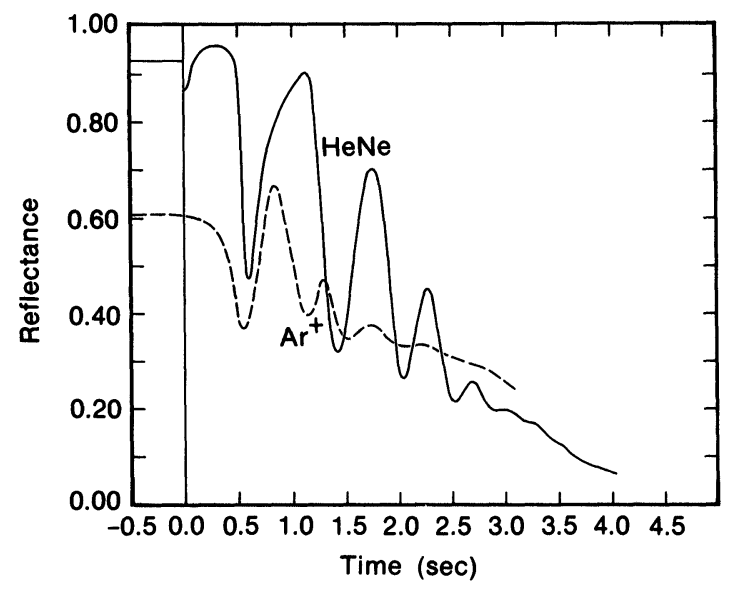

Figure 1 (a) Schematic of the experimental arrangement used for monitoring laser thermal oxidation of copper by optical reflectance. The beam driving the oxidation of the copper film (index $n_{3}$ ) is focussed to a $160 \mu \mathrm{m}$ spot at the surface. The probe beam, focussed to a $25 \mu \mathrm{m}$ spot at the center of the driving beam spot and directed $7^{\circ}$ off normal incidence, undergoes interference within the growing oxide layer (index $n_{2}$ ). The $\mathrm{O}_{2}$ ambient is at 150 Torr. (b) Typical reflectance data for $\mathrm{Ar}^{+}$and $\mathrm{HeNe}$ probe beams. The vertical bar indicates the start of illumination by the driving beam (incident power $=3.3 \mathrm{~W}$ ). These data are very similar to those for $2.2 \mathrm{~W}$ and $4.4 \mathrm{~W}$, save the time scale is longer by a factor of 50 and shorter by a factor of 5 , respectively. 
maxima and thickness in order to obtain a direct measure of the oxidation rate over a range of experimental conditions. If this were a valid approach the slope of an Arrhenius plot made from the data should be independent of which set of minima or maxima in the reflectance curves is used. In fact, this expectation is not borne out, ${ }^{19}$ indicating that the intensity of the reflected light is not simply proportional to the extent of reaction. As will be seen below, this is a consequence of the non-isothermal nature of the laser oxidation process.

Accordingly, a somewhat different approach, computer simulation of the optical reflectance data, was used to obtain information on the oxidation reaction from the experimental results. The following algorithm treats explicitly laser heating of the copper-copper oxide films. For each time interval:

(i) The thicknesses of the copper and copper oxide layers are calculated.

(ii) Based on these thicknesses, and the current value of the temperature-dependent optical constants for the system, the amount of light absorbed from the driving beam is calculated, as are the reflectances of the probe beams.

(iii) The temperature rise resulting from the absorbed energy is determined. The most accurate simulations are obtained when the system is treated as a thin skin of a good conductor on a thermal insulator, a reasonable approximation for copper on sapphire. The temperature dependence of the thermal conductivities of the materials in the system is explicitly included.

(iv) Knowing the temperature, the instantaneous rate of oxidation at an oxygen pressure of 150 Torr is calculated, using rate coefficients determined for isothermal oxidation in a furnace.

(v) After checking the changes in temperature, absorbance and oxidation rate for convergence, return to step (i).

The results of the calculations are shown in Figure 2 for a driving beam power of $4.4 \mathrm{~W}$. The calculated reflectances in Figure $2 \mathrm{a}$ are in excellent agreement with experiment ${ }^{19}$ (cf. Figure $1 \mathrm{~b}$ ), particularly in view of the fact that no adjustable parameters were used in the calculation and that the literature values used for the physical proper- 
(a)

(b)

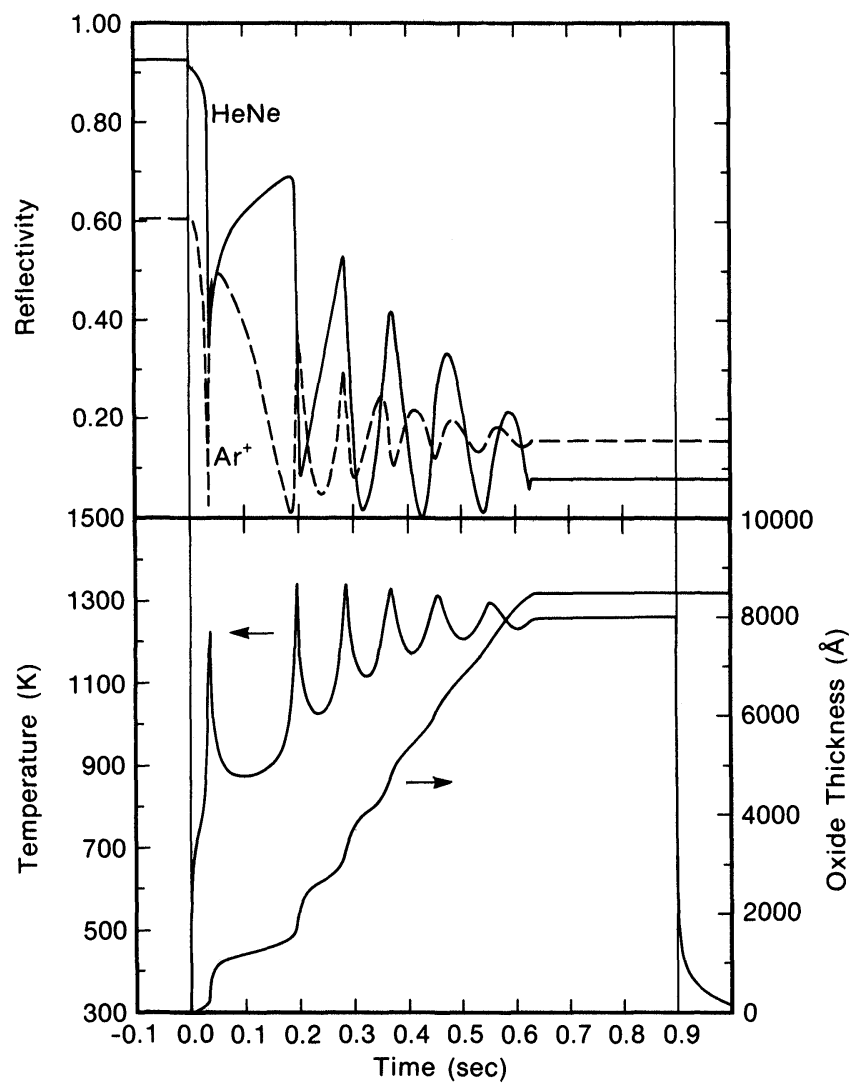

Figure 2 Theoretical simulation of laser oxidation of a copper film. (a) Calculated reflectance of $\mathrm{HeNe}$ and $\mathrm{Ar}^{+}$laser probe beams as a function of time. (b) Corresponding calculations of film thickness and temperature.

ties of the system were largely obtained for bulk, not thin film samples, and were in some cases not well established.

As shown in Figure $2 b$, the model makes two specific predictions concerning the oxide growth process. The first is that the film thickness does not increase in a monotonic way, but in a sequence of steps. This is in stark contrast to classical diffusion-limited growth of cuprous oxide, which obeys kinetics of the form $x \propto \sqrt{ } t$ where $x$ is thickness and $t$ is time. ${ }^{21}$ Second, the calculated temperature does not change in a simple manner, but undergoes strong oscillations over a range of 
$300 \mathrm{~K}$. These predictions, and thus the accuracy of the model itself, were verified experimentally by studying films grown by a sequence of increasing periods of laser irradiation. The thicknesses of the films were measured by depth profiling, and, as seen in Figure 3, the stepwise growth pattern is clear. The existence of temperature spikes and their correlation with destructive interference in the growing oxide layer was demonstrated by exploiting the fact that the reflectivity of the copper film is highly dependent on temperature. ${ }^{19}$

Assured that the theoretical model is realistic, it is possible to understand how the oxidation process works at visible wavelengths. As the oxide film thickness increases, conditions for destructive interference are met and the fraction of light absorbed approaches a maximum. The system temperature rises rapidly, as does the oxidation rate. This cannot be sustained indefinitely since at some point in time the oxide layer will be too thick to support destructive interference, and the absorbed light intensity, the temperature and the oxidation rate will all decline. The drop in the latter is precipitous because of the exponential dependence of the rate on temperature, leading to stepwise growth of the oxide layer as observed. The reduced growth rate persists until absorption begins to increase, and the cycle starts anew. It is not noteworthy that the very rapid rate of oxidation observed experimentally is predicted by the model using furnace oxidation data from the literature. Although there was no reason to believe a priori

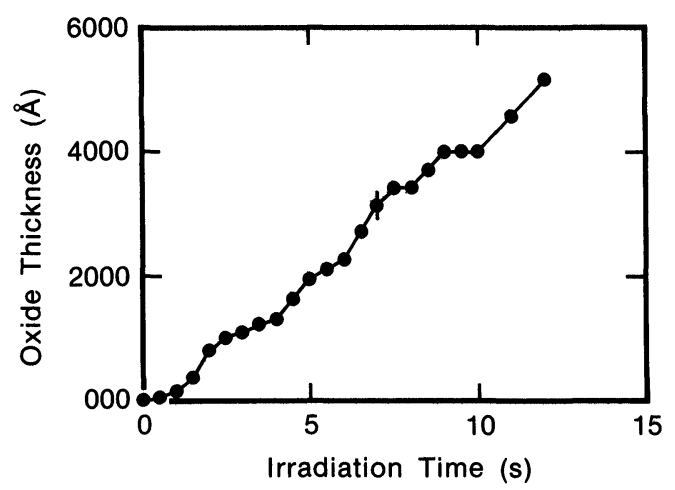

Figure 3 Spot thickness as a function of exposure time measured by Ar ion etching, scanning Auger microscopy and scanning electron microscopy. The incident laser power was $2.8 \mathrm{~W}$. 
that the coefficients and form of the rate law should be the same for furnace and laser oxidation, the good agreement between theory and experiment indicates that the rate determining steps of the two modes of oxidation are likely to be quite similar. This result casts doubt on previous speculations concerning possible special aspects to laser oxidation, and suggests that it is a rather straightforward process: the rapid rate of laser oxidation is seen to be primarily an optical, rather than a chemical or mechanical effect.

\section{SCATTERING AND CHANNELLING OF LIGHT}

More subtle, dynamic optical effects can also strongly influence a laser-driven reaction at the surface. They are mediated primarily by the topography of the laser-modified surface, and can reinforce propensities toward certain topographic features such as straight sidewalls, spikes and gratings. The spatial distribution of light over the modified surface can differ significantly from that of the incident beam, and local reaction rates and chemistry will vary accordingly.

Perhaps the simplest process is waveguiding during etching, in which light is reflected downward by the walls of the etched region. This leads to uniform profiles despite a considerable divergence of the laser beam beyond the focal plane. ${ }^{13,22,23}$ Chemical as well as structural characteristics can be important as they can serve to reinforce waveguiding properties of particular features. Such an effect has been invoked for etching of trenches in CdTe, where preferential desorption of $\mathrm{Cd}$ leaves Te-rich sidewalls. ${ }^{22}$

The importance of chemical effects is particularly evident in laser deposition onto gratings. Such structures often arise spontaneously in photochemical deposition when polarized laser beams are used and surface photolysis (rather than gas phase photolysis) dominates the film growth process. ${ }^{24}$ Scattering of light into surface modes by rough features, e.g. crystallites of a nucleating film, and interference of the scattered light with the incoming beam leads to a spatially inhomogeneous electromagnetic field at the surface. The light intensity distribution is in the form of a grating oriented perpendicular to the polarization vector, leading to deposition in a grating pattern. Once initiated, the grating structure continues to grow via an optical feedback mechanism, in which further coupling of light into surface modes 
is mediated by the grating structure itself. ${ }^{24}$ The surface mode excited depends on the film composition. If it is highly ordered or metallic surface plasma waves can be involved. The grating can also support higher order electromagnetic field structures, as demonstrated by deposition of a grating of half period onto a pre-existing grating using stimulated second order surface plasma wave scattering. ${ }^{25}$ Inverting the process, enhanced growth of a film deposited photochemically using a beam patterned into a holographic grating occurs for those grating spacings where coupling of light into surface plasma waves is resonant. ${ }^{26}$ Similarly, the profile of a holographic grating photochemically etched into $p$-type GaAs shows evidence for modification of the light intensity at the surface by interaction of the grating with the incident laser beam. ${ }^{27}$

Because the result of interaction of light with a periodically structured surface is a spatially inhomogeneous electromagnetic field, any intensity-dependent photoprocesses taking place at the surface will also be spatially inhomogeneous. As noted above, the intensity dependence of the decomposition rate leads to the grating structure of the film. If the chemistry itself is intensity dependent, then the films can also be spatially inhomogeneous in chemical composition and even morphology, depending on the mixture of elements. ${ }^{28,29}$ Such inhomogeneities can provide a great deal of information on the photodissociation mechanism at the surface that might be otherwise unobtainable because of the very small film sizes (typically a few hundred $\mu \mathrm{m}^{2}$ or less) and numbers of surface species. The complexity which can arise is illustrated by the photochemical deposition of carbon-contaminated copper films using a polarized continuous $257 \mathrm{~nm}$ laser beam. ${ }^{28,29}$ The gas phase organometallic precursor used for film growth is bis$\left(1,1,1,5,5,5\right.$-hexafluoropentanedionate) copper (II), or Cu(hfac) ${ }_{2}{ }^{29,30}$ Its vapor pressure is sufficiently low that film growth involves mainly surface, not gas phase, photolysis. Excitation of the copper complex in the vicinity of $250 \mathrm{~nm}$ causes an internal charge transfer to occur, most likely leading to formation of a copper (I) adduct and an acetylacetonyl free radical on the surface of the film. The adduct eventually dissociates to form zero valent metal. The ligands decompose to form volatile products containing $\mathrm{C}, \mathrm{O}$ and $\mathrm{F}$, and involatile products containing $\mathrm{C}$. The relative amounts of $\mathrm{C}$ and $\mathrm{Cu}$ in the films were measured by scanning Auger microscopy (SAM), and were found to depend sensitively on incident laser beam intensity, among other 
factors. ${ }^{29}$ A strong increase in copper concentration is found among films deposited with average intensities from $10^{3}-10^{4} \mathrm{~W} / \mathrm{cm}^{2}$. A radial decrease in copper concentration is also observed on individual films because of the Gaussian intensity profile of the laser beam.

Transmission electron (TEM) micrographs of a typical film are shown in Figure 4. The grating structure is clearly evident, as is a periodicity in film morphology. The thicker parts of the ripples are amorphous, and the ripple minima have narrow rows of more ordered grains about $200 \AA$ in diameter. These grains are not metallic, but appear to be a separate phase of the carbon-copper composite comprising the film. This observation suggests that the films are not homogeneous in composition. This possibility is also indicated by the strong dependence of composition on light intensity. SAM with a spatial resolution of $500 \AA$ was used to measure carbon and copper concentrations across ripples varying from $1000-3000 \AA$ in width, and also across much larger structures arising from beam diffraction.

(a)

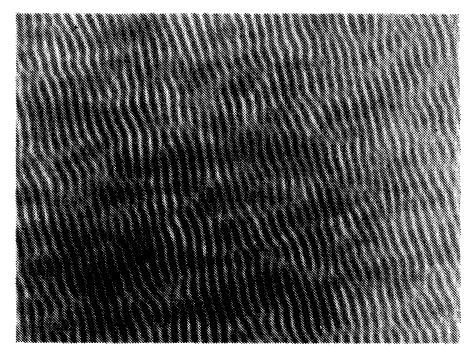

(b)

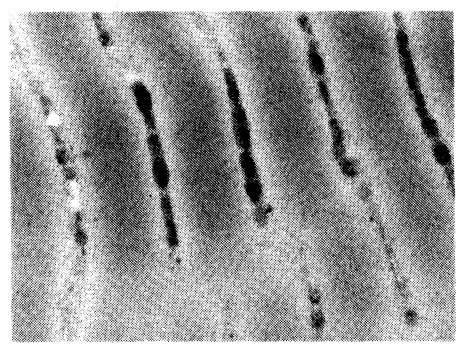

Figure 4 Transmission electron micrographs of a photochemically deposited copper film. (a) 11,000 X magnification, (b) 100,000 X magnification, blow-up of a region near the center of the film (lower left hand portion of (a)). The ripple spacings are approximately $1800 \AA$. 
Strong sinusoidal variations are found, with the oscillations in $\mathrm{C}$ and $\mathrm{Cu}$ signals being approximately $180^{\circ}$ out of phase. Typical data are shown in Figure 5. The maxima in copper content are found at the peaks of the ripples, and the minima at the valleys. This correlates well with the observation that copper concentration increases with increasing light intensity. Since it is extremely unlikely that significant temperature gradients on a scale of $1000 \AA$ exist in these films, the SAM data provide strong evidence that photolysis of the acetylacetonyl ligands is responsible for their dissociation on the surface, and that increasing light intensity promotes formation of volatile photoproducts.

Thus far, we have discussed the effect of the surface electromagnetic field on the film. This does not address the fact, however, that it is the structure of the film which locks in a particular field structure. The scattering process leading to coupling of light into surface waves is enhanced by near-resonance with some excitation in the growing film. Such excitations are in turn dependent on the exact local composition

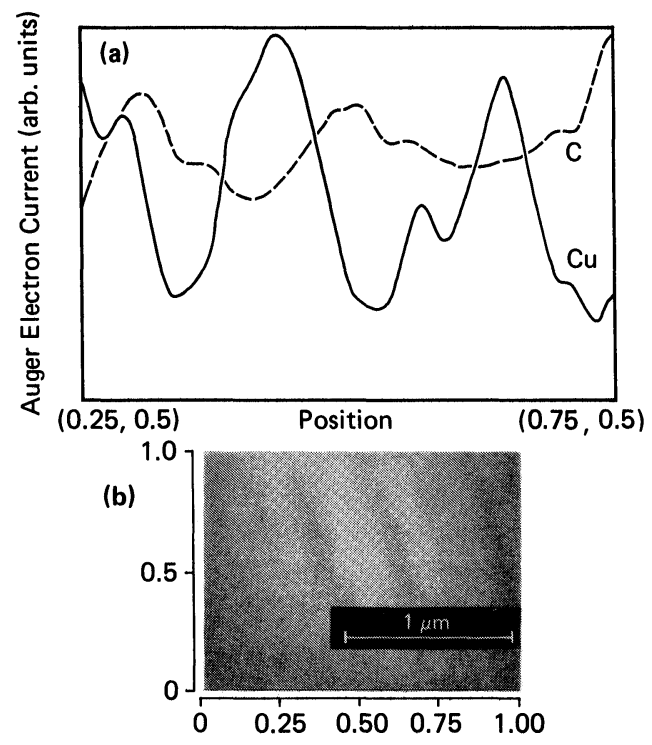

Figure 5 (a) Carbon and copper compositions measured while scanning across ripples shown in (b). The incident laser power was $3.5 \mathrm{~mW}$. The modulation depth for both the $\mathrm{C}$ and $\mathrm{Cu}$ signals is about $10 \%$. 
of the film since the scattering process is local: variations in excitation energy are indicated by variations in ripple spacing. TEM and SAM data show that as the average copper content of the films increases, the spacing of ripples decreases from $1800 \AA$ to $1100 \AA$, corresponding to development of metallic, or plasmon, character to the excitation. It is evident, therefore, that the spatial inhomogeneities of the film and the electromagnetic field at the surface cannot be considered independently of one another.

Ripple formation is characterized by structures running perpendicular to the direction of the polarization vector of the laser beam. Additional electromaganetic field effects can be found parallel to the polarization, due to the high fields which develop at absorbing features of high radius of curvature. Rather little work has been done in this area, but the influence of fields on absorptivity and relaxation processes may be important and pervasive. It has been shown for example, that $\mathrm{Cd}$ photochemically deposited onto silver and cadmium spheres grows preferentially along the polarization of the beam, forming ellipsoidal structures. ${ }^{31}$ The feedback mechanism in such a situation is evident - the field strengths will always be highest as the tips of the elongated structures, promoting continued preferential growth along that direction. An analysis of the influence of simple prototypical geometries on scattered electromagnetic field strengths has been made. 32

\section{SUMMARY}

The real-time evolution of reactions driven by a laser beam incident on the surface typically involves an intricate assembly of chemical, optical and thermal components which must be understood as a whole. It cannot be assumed that isothermal, steady state chemical kinetics predominate at the surface, nor can the light intensity distribution driving the chemistry be automatically taken to be a simple function of time and position. Simple models such as Arrhenius plots should be used with extreme caution for this reason. The most successful theoretical treatments of laser-driven surface reactions simulate a progression of events explicitly, calculating changes in all significant quantities iteratively. This is a brute force approach, but the only accurate one at present since there are not enough data to develop 
simpler analytical models having broad applicability. Indeed, it is doubtful that all key types of interdependences have even been identified at this time, and several can be operative at once. This aspect of laser-driven surface chemistry, uniting elements of physics, optics and chemistry, is probably the most interesting and challenging of all.

\section{Acknowledgements}

It is a pleasure to thank my collaborators R. J. Wilson, L. Baufay and T. Baum, who have made important contributions to many of the ideas discussed here.

\section{References}

1. R. M. Osgood, Mater. Res. Soc. Symp. Proc. 75, 3 (1987).

2. R. M. Osgood and H. H. Gilgen, Ann. Rev. Mater. Sci. 15, 549 (1985).

3. Y. Rytz-Froidevaux, R. P. Salathe and H. H. Gilgen, Appl. Phys. A37, 121 (1985).

4. F. A. Houle, Appl. Phys. A41, 315 (1986).

5. D. J. Ehrlich, Solid State Technol., December, 1985, pp. 81-85.

6. D. J. Ehrlich and J. Y. Tsao, J. Vac. Sci. Technol. B1, 969 (1983).

7. S. D. Allen and A. D. Trigubo, J. Appl. Phys. 54, 1641 (1983).

8. S. D. Allen, R. Y. Jan, S. M. Mazuk and S. D. Vernon, J. Appl. Phys. 58, 327 (1985).

9. S. D. Allen, J. A. Goldstone, J. P. Stone and R. Y. Jan, J. Appl. Phys. 59, 1653 (1986).

10. W. M. Grossman and M. Karnezos, J. Vac. Sci. Technol. B5, 843 (1987).

11. G. M. Davis and M. C. Gower, J. Appl. Phys. 61, 2090 (1987).

12. G. Koren, Appl. Phys. Lett. 50, 1030 (1987).

13. B. Braren and R. Srinivasan, J. Vac. Sci. Technol. B3, 913 (1985).

14. K. Piglmayer, J. Doppelbauer and D. Bauerle, Mater. Res. Soc. Symp. Proc. 29, 47 (1984).

15. Y. C. Du, U. Kempfer, K. Piglmayer, D. Bauerle and U. M. Titulaer, Appl. Phys. A39, 167 (1986).

16. R. B. Jackman, J. S. Foord, A. E. Adams and M. L. Lloyd, J. Appl. Phys. 59, 2031 (1986).

17. L. Baufay and M. E. Gross, Mater. Res. Soc. Symp. Proc. 101, 89 (1988).

18. M. E. Gross, A. Applebaum and K. J. Schnoes, J. Appl. Phys. 60, 529 (1986).

19. L. Baufay, F. A. Houle and R. J. Wilson, J. Appl. Phys. 61, 4640 (1987).

20. L. Baufay, F. A. Houle and R. J. Wilson, Mater. Res. Soc. Symp. Proc. 75, 281 (1987).

21. See, for example, K. Hauffe, "Oxidation of Metals," Plenum, New York, 1965.

22. C. Arnone, M. Rothschild and D. J. Ehrlich, Appl. Phys. Lett. 48, 736 (1986).

23. D. Podlesnik, H. H. Gilgen and R. M. Osgood, Jr., Appl. Phys. Lett. 48, 496 (1986).

24. S. R. J. Brueck and D. J. Ehrlich, Phys. Rev. Lett. 48, 1678 (1982).

25. D. J. Ehrlich and S. R. J. Brueck, Appl. Phys. Lett. 47, 216 (1985).

26. C. J. Chen, H. H. Gilgen and R. M. Osgood, Jr., Opt. Lett. 10, 173 (1985).

27. R. Matz, Mater. Res. Soc. Symp. Proc. 75, 657 (1987).

28. R. J. Wilson and F. A. Houle, Phys. Rev. Lett. 55, 2184 (1985).

29. F. A. Houle, R. J. Wilson and T. H. Baum, J. Vac. Sci. Technol. A4, 2452 (1986). 
30. C. R. Jones, F. A. Houle, C. A. Kovac and T. H. Baum, Appl. Phys. Lett. 46, 97 (1985).

31. C. J. Chen and R. M. Osgood, Jr., Phys. Rev. Lett. 50, 1705 (1983).

32. S. R. J. Brueck, D. J. Ehrlich, D. V. Murphy and J. Y. Tsao, Mater. Res. Soc. Symp. Proc. 29, 295 (1984). 